\title{
A Contactless loT based Intelligent Parking Solution for Smart Cities
}

\author{
Dhairya Parekh \\ Department of Information \\ Technology \\ K. J. Somaiya College of \\ Engineering, India
}

\author{
Eeshan Mundhe \\ Department of Information \\ Technology \\ K. J. Somaiya College of \\ Engineering, India
}

\author{
Jigar Jain \\ Department of Information \\ Technology \\ K. J. Somaiya College of \\ Engineering, India
}

\begin{abstract}
With the advent of this century, automobiles have introduced a remarkable comfort in our lives but a grave concern of parking surely follows. The 'new normal' that the COVID-19 pandemic has introduced, requires us to eliminate all unnecessary contact to contain the spread of the virus. Hence, more than ever before, it is now quintessential for smart cities to have robust parking solutions equipped with technologies that allow this activity to be convenient for everyone. The authors in this paper have proposed a smart, IoT based, reliable, and affordable parking solution for smart city planners and developers. The mechanism is designed to keep all essential components such as network infrastructure and secure exchange of information in focus to also answer all the challenges that such a solution might face during operations.
\end{abstract}

\section{General Terms}

Smart City, IoT, Mobile Application

\section{Keywords}

IoT, Contactless Parking, IR Distance Sensor, Network Infrastructure, Data \& Security, Quick Response (QR) Code, Mobile Application

\section{INTRODUCTION}

Smart City infrastructure designers plan automotive behaviour with a lot of detailing and acumen. Transportation indeed is a major concern for policy makers. With a growing road network and changing demographics pattern, individuals today often make use of their personal vehicles. This usage draws attention to the requirement of adequate parking facilities. Major Indian cities already face an issue while managing their parking infrastructure because of underdeveloped solutions. Moreover, even if they do have a solution in place, it is often expensive to install at other locations and maintain the same. As a result, it degrades with time and the expenditure becomes a total waste. The authors in this paper explain a mechanism for a low cost, easy to deploy and maintain, reliable, secure, IoT based end-to-end intelligent solution for parking lots.

GPS technology enables users to find an optimal route for traveling to a particular destination, but it does not help us in finding an empty parking spot nearby when one arrives at their destination. Users usually have to search for an empty parking spot by themselves, which involves human interaction and contact.

The paper proposes a mechanism that limits the need for human contact by making the use of infrared distance sensors, a resource-constrained network infrastructure, Quick Response (QR) code for user identification and a mobile application that facilitates digital payments and hosts all the service modules of the mechanism. The data generated or required during the operation is stored and made available with the help of cloud-based databases. The stated components are explained in section III. The relevant data can be accessed by an authenticated and registered user to check the availability of empty parking spots in the nearby lot. The cloud database gets updated in real-time as a user parks the vehicle or checks out, so other users can get updated status of the parking lot immediately.

The solution takes into consideration the cost factor as well as the privacy and security of users to provide a hassle-free process. Everything from finding a parking spot to paying for the occupation of the spot while exiting the parking can be done without contact, virtually, to ensure the user does not have to come into contact with anyone/anything.

The paper is structured in a way that section II states the existing literature in relation to digitized parking lots. All the major components of the mechanism are listed and explained in section III. Section IV gives clarity for the implementation of the mechanism. Section V explains the discussions appropriate to the paper, while section VI elaborates on the future scope of stated research before providing a conclusion to this paper.

\section{LITERATURE REVIEW}

V. U. et.al in [1] describe their IoT based solution to handle parking slot availability for a smart city. Authors have illustrated how data can be gathered using cost-effective ultrasonic sensors and is sent to a Firebase cloud for processing. For making this information available to users, the authors have stated the use of an android application. It discusses the importance of hardware costs for deployment and maintenance while eliminating unnecessary use of devices or components for the stated purpose. In [2], authors have used an RFID based solution for vehicle identification. However, it uses a much cost extensive method for the stated purpose prone to damage and deterioration. Furthermore, the authors fail to provide a real-time count for the occupied slots as it just factors vehicular movement in and out of a parking lot which has limitations. The IoT infrastructure has a resemblance to the model stated in [1] when it comes to storing collected data on the cloud.

The authors in [3] have taken focus to discuss a resourceconstrained network design for IoT devices. A comparison of network algorithms has been made. It explains a practical scenario of how a low-cost IoT device can be deployed with sensors and stand-alone networking devices at the same time. The authors have also discussed an efficient setting for data communication over the same network. K. B. Dsouza, et.al in [4] have mentioned the use of existing infrastructure like 
CCTVs for image processing using ML algorithms. However, the authors have based their solution on a lot of assumptions that blow up in a practical setting. The methodology is both expensive and difficult to maintain given the fact that it indeed has less reliability than the solutions in [1] or [3]. In [5], authors have explained a parking management system that facilitates ease of use and implementation of their system. It covers actions like searching for a parking slot or reserving in some cases to the occupation of that slot, payments, and notifications for this entire process cycle. The authors have also stated their use of data that the system collects for analytical reporting purposes.

In [6], the authors suggest using Kalman Filters for finding a parking spot by investigating the suitability of different sized taxi fleets to check availability for on-street parking using the crowd sensing technique. Authors have performed analyses for the spatiotemporal sensing coverage for the purpose that could be achieved by taxi fleets of varied groups, in a realtime large urban set-up and compared the results with the one obtained by stationary sensors. The benefits of using Kalman filters which is a Machine Learning approach is understood as it concludes that crowd-sensing is a great alternative to static parking sensors. However, this is not a viable approach as it would require a lot of data for training the Machine Learning model.

In [7] the authors have described an IoT-based solution for parking in a structured environment. The solution is implemented using advanced Honeywell sensors, and the unoccupied parking spots are illuminated with lights so they can be located easily. Their system is fully automatic and reduces manpower involved in the process of finding a parking spot.

\section{COMPONENTS OF THE PROPOSED MECHANISMS}

\subsection{Hardware Sensor Component for Parking Slot}

First and foremost, the mechanism needs a way to identify and report to the system that a particular parking spot is vacant. For this, each sensor node will be given a unique onebyte alphanumeric addressing system. The sensor will send either 0 (not occupied) or 1 (occupied) bit of data for reporting occupancy status over a network infrastructure discussed in the next subsection along with the parking spot's location. The mechanism endorses the use of IR distance sensor module because of its varied capabilities. It is an ideal sensor for complex surfaces with minimal sensitivity to external conditions. It's a low cost, low maintenance module unit suitable for the intended operations with easy scalability. In table 1, a comparison of different sensors modules that were studied by the authors to make this endorsement. Factors such as range for sensing, ability to use for complex surfaces, sensitivity to external factors, cost of operation (initial and maintenance cost) have been taken into consideration. Exclusion of technology with more capabilities than required for the operation has been of primary concern so as to have a cost-effective practical solution.
Table 1 Comparison of Sensors for Hardware Sensor Component

\begin{tabular}{|c|c|c|c|c|}
\hline $\begin{array}{l}\text { Factors/ } \\
\text { Sensors }\end{array}$ & $\begin{array}{c}\text { IR } \\
\text { Distance } \\
\text { Sensor }\end{array}$ & $\begin{array}{c}\text { Ultraso } \\
\text { nic } \\
\text { Sensor }\end{array}$ & $\begin{array}{l}\text { LiDAR } \\
\text { Sensors }\end{array}$ & $\begin{array}{c}\text { LED } \\
\text { Time-of- } \\
\text { Flight } \\
\text { Distance } \\
\text { Sensor }\end{array}$ \\
\hline Range & Low & Low & High & Moderate \\
\hline $\begin{array}{l}\text { Ability to } \\
\text { use for } \\
\text { complex } \\
\text { surfaces }\end{array}$ & Yes & No & Yes & Yes \\
\hline $\begin{array}{c}\text { Sensitivity } \\
\text { to external } \\
\text { factors }\end{array}$ & No & Yes & No & No \\
\hline $\begin{array}{l}\text { Risk of } \\
\text { Harm }\end{array}$ & Low & Low & $\begin{array}{c}\text { Moderat } \\
\mathrm{e}\end{array}$ & Low \\
\hline $\begin{array}{c}\text { Cost of } \\
\text { Operation }\end{array}$ & Low & Low & High & High \\
\hline
\end{tabular}

Understanding table. 1: LiDAR or LED Time of Flight Distance Sensor has a better range than IR Distance Sensor but the range that later delivers is sufficient for the purpose. IR Distance Sensor has the ability to detect complex surfaces amongst low-cost sensor modules. The ultrasonic sensor's reading is impacted because of external factors that are undesirable. LiDAR may be harmful to humans under certain conditions which makes it less suitable for the intended use. With overall comparison including the cost of operation, IR Distance Sensor offers the best solution amongst the sensors studied.

\subsection{Network Infrastructure Component for Communication}

It is quintessential for the proposed mechanism to have a network infrastructure that is reliable, efficient, and is cheaper to use in a resource-constrained setup. It is expensive for each sensor node to communicate independently to the cloud server (which is the central data storage) and therefore it will follow a linear mapped path. Each node will communicate with a central parent node (CPN) in the facility and that CPN will push data to the cloud. This data exchange will take place using a User Datagram Protocol (UDP). To ensure that the data is correctly received by the CPN, it will send an acknowledgment to the sensor node. The central parent node will have Fiber Optic Architecture with a connection using Local Area Networks (LAN). This methodology also eliminates connectivity issues for underground parking structures or locations with connectivity challenges. With this arrangement, the mechanism is also equipped with handling an expansion of service.

\subsection{Data Security and Storage Component}

The proposed system consists of data from the parking structure as well as user information. The proposed mechanism will involve storage and transfer of sensitive user data; hence it is necessary to ensure the security of this data from security breaches. The authors also want the data to be handled in an optimal way in order to get the results of a 
query as quickly as possible. Therefore, the management of storage and security of the data can be understood using the following steps:

1. Data Storage: The mechanism will use the cloud version of the SQL database for storing both the user data as well as the parking data. This is because the databases can be scaled easily and cheaply, while the data remains secure and easy to access.

2. Data security: The mechanism uses end-to-end encryption to prevent security breaches from stealing the information. The application will also have an access control mechanism to increase the security of the data by granting access to users only on valid devices, and by giving access only after verification of passcode from the user. For achieving the end to end communication security, the mechanism will use the Secure Socket Layer Protocol or Transport Layer Security Protocol.

\subsection{Quick Response (QR) Code Component}

The mechanism will require a lot of random codes as every vehicle will scan a unique $\mathrm{QR}$ code and as soon as the vehicle enters or exits the parking lot, a new QR code will be generated for the next vehicle. These codes should not be easy to guess, so the mechanism will generate random codes and encode them into QR codes. This will enable the user to scan the $\mathrm{QR}$ codes easily using the mobile application. While exiting, the user will scan a different $\mathrm{QR}$ code through the application, so once a user uses the QR code at entry, there is no need to store it in the system.

\subsection{UI Component for Mobile Application}

The UI component integrates the usage of all the aforementioned components. The user will be able to scan a secured quick response code (QR code) while entering the parking lot using the mobile application. The user's authenticated profile which is accessible through the mobile application gets checked-into the server which stores the timestamp when the user arrives at the structure. The slot where they park also gets updated to the cloud server so that the user can remember where they parked as it's easy to relocate their vehicle when they want to leave. Later, when the user releases their occupation of the parking slot and exits the parking lot, they can scan yet another QR code on exit. This time the timestamp of exit is sent to the cloud server and occupation charges are calculated accordingly. The mobile application will also provide different commonly used payment options for the user to choose from to complete the payment. The user can look at their transaction history or check for a free spot before arriving at the parking structure using this application.

\section{IMPLEMENTATION}

Users can use the mobile application to register with their personal details and their vehicle's registration number. After the details are verified by the system, the user is eligible to use the application. The payment wallet can be loaded with value but this action is completely optional.

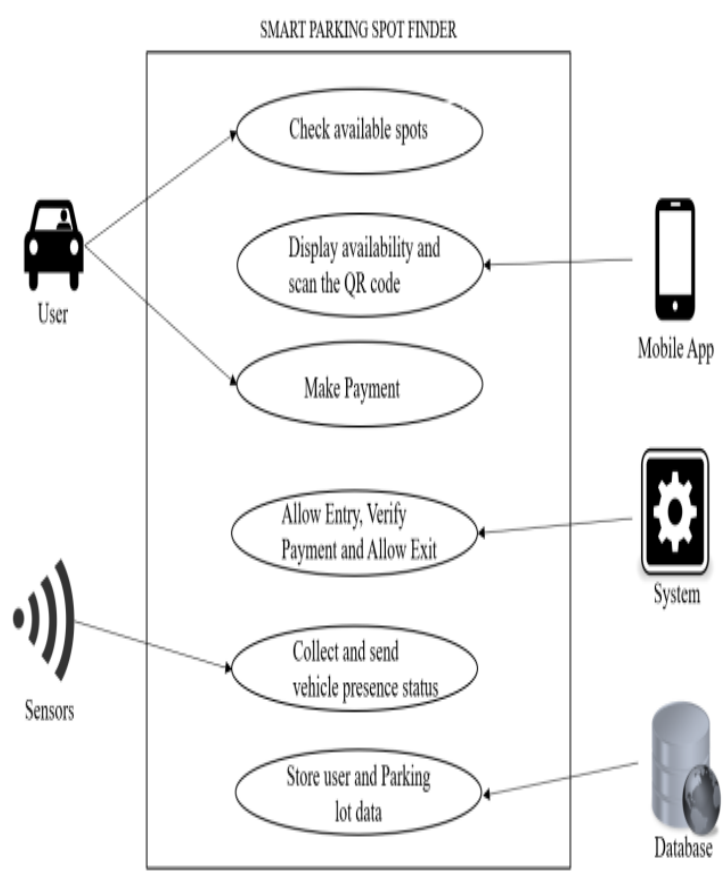

Fig. 1 Use Case Diagram of Proposed Mechanism

When the user identifies a vacant parking spot through the application and arrives at the entrance (or the In-gate) of the parking lot, the user will request a $\mathrm{QR}$ code through the app for entry. A unique $\mathrm{QR}$ code will be displayed on the screen at the entrance which the user has to scan using the mobile application. This QR code will verify the user details as well as insert the vehicle number in the system and will allow the user to enter the parking lot. After the user enters the parking lot, the screen displaying the QR code will refresh so that the next user can generate a new QR code.

When the user parks the vehicle in a particular spot, the infrared sensor at that spot will record the presence of the vehicle and update the database with the parked vehicle's

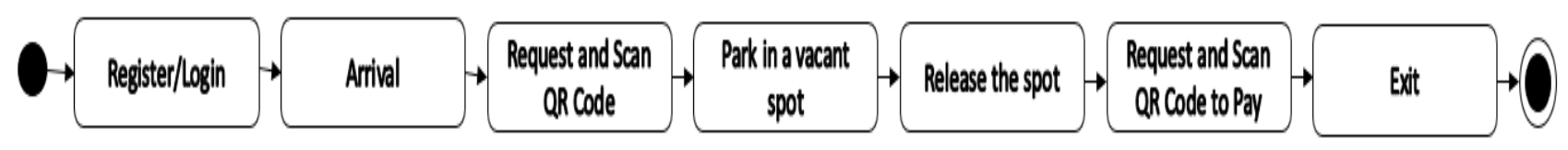

Fig. 2: Activity Diagram for a User in the Proposed Mechanism

position and the parking slot number. This will help the user to find the vehicle using the mobile application in case they forget where they had parked earlier by following the parking slots which are sequentially numbered.

While exiting, the user will drive near the exit point where a new $\mathrm{QR}$ code has to be generated for verifying that the user has paid the parking charges for the entire duration of occupation of the parking spot. The payment can be done through the application, and upon meeting the requirements, the user will be allowed to exit. The infrared sensors will ensure that only 1 vehicle enters and exits with one QR code. 
The UI of the application will be user friendly so that the users do not have to face any hurdles while searching or paying for the parking. There will be an option to view transaction history, locate nearby available parking spaces, make the payment, and edit profile. All edge cases, in case they occur, will be dealt with remote human assistance. Figure 1 illustrates the Use Case Diagram of the proposed mechanism, while figure 2 illustrates the Activity Diagram for any User in the Proposed Mechanism.

\section{RESULTS AND DISCUSSION}

As city administrations around the globe continue to allocate larger budgets for smart city projects, the use of IoT-based intelligent solutions will rise undoubtedly. The global market for smart parking infrastructure is projected to reach $\$ 11.5$ billion by 2025 [16]. The top impacting factors that influence this growth are: rise in investments and initiatives by government, rise in demand for IoT based technology, and rise in the number of vehicles on the streets. With constructive steps to address efficient data handling and security management in place, the robustness of the solution is amplified manifold. With use of the proposed mechanism, city planners and developers can provide convenience and ensure safety for their citizens.

\section{FUTURE WORK}

RFID technology is generally considered quite reliable and convenient to use. Given this, it is actually expensive to employ such a mechanism if it has to start from the installation of these RFID cards in vehicles. In India, FASTag is one such deployment of RFID based solutions. With correct settings that could be integrated to achieve a true contactless and for faster transaction process instead of the $\mathrm{QR}$ code module of the current mechanism. The mechanism will also generate a lot of data about the infrastructure usage which can be used to perform analytics to further optimize the proposed mechanism. In many cities there are not enough parking lots; hence parking on the designated street is allowed where it's feasible. Currently, the system is designed for a typical parking lot, but with some modifications, the system can also be designed for extended use.

\section{CONCLUSION}

The proposed solution provides an efficient and fully digital way of performing tedious tasks. This reduces human effort and makes the process of parking quick, inexpensive, easy, reliable and maintainable. The user-friendly mobile application provides the users with a convenient facility to find parking spots even to those with limited technology exposure. The proposed mechanism being fully digital and contactless, removes the need for human interaction, thus adding pandemic proofing to this usage. The authors believe, that their proposed mechanism not only ensures the efficacy of IoT technology but also provides a complete design for smart city planners.

\section{REFERENCES}

[1] V. U., S. Sharma, B. Katiyar and Y. Prashanth, "A Wireless Sensor Node Based Efficient Parking Slot Availability Detection System For Smart Cities," 2018 Recent Advances on Engineering, Technology and Computational Sciences (RAETCS), Allahabad, 2018, pp. 1-6, doi: 10.1109/RAETCS.2018.8443892.

[2] P. M. Akshay, K. Murugesh and Y. Patra, "IoT based Automated Paid Parking Using Electromagnetic RFID Tag," 2019 International Conference on Intelligent Computing and Control Systems (ICCS), Madurai, India,
2019 pp.

$1451-1452$

doi:

10.1109/ICCS45141.2019.9065837.

[3] G. Şimşek and M. T. Sandıkkaya, "Parking IoT: An IoT Architecture to Collect Availability Data from Parking Lots," 2020 9th Mediterranean Conference on Embedded Computing (MECO), Budva, Montenegro, 2020, pp. 1-5, doi: 10.1109/MECO49872.2020.9134335.

[4] K. B. Dsouza, S. Mohammed and Y. Hussain, "Smart parking - An integrated solution for an urban setting," 2017 2nd International Conference for Convergence in Technology (I2CT), Mumbai, 2017, pp. 174-177, doi: 10.1109/I2CT.2017.8226115.

[5] N. Farooqi, S. Alshehri, S. Nollily, L. Najmi, G. Alqurashi and A. Alrashedi, "UParking: Developing a Smart Parking Management System Using the Internet of Things," 2019 Sixth HCT Information Technology Trends (ITT), Ras Al Khaimah, United Arab Emirates, 2019, pp. 214-218, doi: 10.1109/ITT48889.2019.9075113.

[6] F. Bock, S. Di Martino and A. Origlia, "Smart Parking: Using a Crowd of Taxis to Sense On-Street Parking Space Availability," in IEEE Transactions on Intelligent Transportation Systems, vol. 21, no. 2, pp. 496-508, Feb. 2020, doi: 10.1109/TITS.2019.2899149.

[7] D. Ashok, A. Tiwari and V. Jirge, "Smart Parking System using IoT Technology," 2020 International Conference on Emerging Trends in Information Technology and Engineering (ic-ETITE), Vellore, India, 2020, pp. 1-7, doi: 10.1109/ic-ETITE47903.2020.457.

[8] R. Kanan and H. Arbess, "An IoT-Based Intelligent System for Real-Time Parking Monitoring and Automatic Billing," 2020 IEEE International Conference on Informatics, IoT, and Enabling Technologies (ICIoT), Doha, Qatar, 2020, pp. 622-626, doi: 10.1109/ICIoT48696.2020.9089589.

[9] M. Muttillo, G. Barile, T. Gabriele and F. F. Donati, "Towards Smart Cities: A Low-Cost Battery Operating Electronic System for Vehicles Smart Parking," 2020 International Conference on Mathematics and Computers in Science and Engineering (MACISE), Madrid, Spain, 2020, pp. 246-249, doi: 10.1109/MACISE49704.2020.00052.

[10] S. P. Patro, P. Patel, M. K. Senapaty, N. Padhy and R. D. Sah, "IoT based Smart Parking System: A Proposed Algorithm and Model," 2020 International Conference on Computer Science, Engineering and Applications (ICCSEA), Gunupur, India, 2020, pp. 1-6, doi: 10.1109/ICCSEA49143.2020.9132923.

[11] M. Kannan, L. William Mary, C. Priya and R. Manikandan, "Towards Smart City through Virtualized and Computerized Car parking System using Arduino in the Internet of Things," 2020 International Conference on Computer Science, Engineering and Applications (ICCSEA), Gunupur, India, 2020, pp. 1-6, doi: 10.1109/ICCSEA49143.2020.9132876.

[12] M. Ramasamy, S. G. Solanki, E. Natarajan and T. M. Keat, "IoT Based Smart Parking System for Large Parking Lot," 2018 IEEE 4th International Symposium in Robotics and Manufacturing Automation (ROMA), Perambalur, Tamil Nadu, India, 2018, pp. 1-4, doi: 10.1109/ROMA46407.2018.8986731. 
[13] C. Ajchariyavanich et al., "Park King: An IoT-based Smart Parking System," 2019 IEEE International Smart Cities Conference (ISC2), Casablanca, Morocco, 2019, pp. 729-734, doi: 10.1109/ISC246665.2019.9071721.

[14] M. Meenaloshini, J. Ilakkiya, P. Sharmila, J. C. Sheffi Malar and S. Nithyasri, "Smart Car Parking System in Smart Cities using IR," 2019 3rd International Conference on Computing and Communications Technologies (ICCCT), Chennai, India, 2019, pp. 178182, doi: 10.1109/ICCCT2.2019.8824953.
[15] P. Solic, R. Colella, L. Catarinucci, T. Perkovic and L. Patrono, "Proof of Presence: Novel Vehicle Detection System," in IEEE Wireless Communications, vol. 26, no 6, pp. 44-49, December 2019, doi: 10.1109/MWC.001.1900133.

[16] Smart Parking Market by Type (Off-street and On-street Parking), Technology (IoT, Ultrasonic, and RFID), Application (Security \& Surveillance, Smart Payment System, E-parking, and License Plate Recognition), and End User (Commercial and Government) - Global Opportunity Analysis and Industry Forecast, 2018-2025 